THE Astrophysical JouRnal, 495:309-316, 1998 March 1

(C) 1998. The American Astronomical Society. All rights reserved. Printed in U.S.A.

\title{
A PROPOSED MODIFICATION OF THE RATE EQUATIONS FOR REACTIONS ON GRAIN SURFACES
}

\author{
P. CASElli \\ Osservatorio Astrofisico di Arcetri, Largo Enrico Fermi 5, I-50125 Firenze, Italy \\ T. I. HASEGAWA \\ Department of Astronomy, Saint Mary's University, Halifax, Nova Scotia, B3H 3C3, Canada \\ AND \\ ERIC HERBST \\ Departments of Physics and Astronomy, Ohio State University, Columbus, Ohio 43210; herbst@mps.ohio-state.edu \\ Received 1996 December 19; accepted 1997 October 10
}

\begin{abstract}
The rate equations currently utilized for grain reactions in gas-grain chemical models of interstellar clouds are inappropriate under certain conditions because of the discrete nature of grain particles. Appropriate modifications are suggested, and calculated surface abundances for various molecules are tested against Monte Carlo calculations for three simple systems. In the first system, the gas is comprised of only $\mathrm{O}$ and $\mathrm{H}$ atoms that accrete on grain surfaces and subsequently react to form only the diatomic molecules $\mathrm{H}_{2}, \mathrm{OH}$, and $\mathrm{O}_{2}$. In the second system, $\mathrm{N}$ atoms are also present in the gas, and the grain chemistry leads to more molecules, including the polyatomic species $\mathrm{NH}_{3}$ and $\mathrm{H}_{2} \mathrm{O}$. In the third and final system, the gas contains $\mathrm{H}_{2}$, and the contribution of this species to grain chemistry is considered. The modifications to the rate equations lead to reasonable agreement with the results of Monte Carlo simulations for all three systems. These modifications can then be used in time-dependent gas-grain chemical models until a more detailed but usable theory becomes available.
\end{abstract}

Subject headings: dust, extinction - ISM: molecules - molecular processes

\section{INTRODUCTION}

At a 1995 March conference on astrochemistry in Leiden, Holland, A. G. G. M. Tielens suggested that the rate equations used to describe grain surface chemistry in chemical models of interstellar clouds are inappropriate because they do not take account adequately of the discrete nature of grains (see also Charnley, Tielens, \& Rodgers 1997). Since a large number of models include this convenient approach (e.g., Pickles \& Williams 1977; Brown \& Charnley 1990; Hasegawa, Herbst, \& Leung 1992; Hasegawa \& Herbst 1993; Shalabiea \& Greenberg 1994), which is so far the only method to make the coupling between gas-phase and grain chemistry quite manageable in time-dependent calculations, Tielens's point is an important one to explore. Rate equations, which equate time derivatives of concentrations to the sum of adsorption, evaporation, and chemical reaction terms, yield average concentrations or numbers of surface particles as functions of time. The concept of averages is adequate if one is dealing with large surfaces containing many reactive particles at any given time. Such averages may be unreliable, however, if the average number of reactive species on a dust particle is less than unity. In this case, the chemistry can approach an "accretion limit," in which successive reactive particles sticking to a particular grain are likely to react with one another if at least one of them migrates on the grain rapidly enough to find the second particle before yet another reactive species adsorbs. Such a limit, and situations in the vicinity of this limit in which rate coefficients play a limited role, can be treated by a Monte Carlo procedure, as was done by Tielens \& Hagen (1982) in a steady state calculation of evolved interstellar grain mantles. In his lecture, Tielens showed that for a simple system, the Monte Carlo procedure leads to results that are very different from the results of rate equations. However, this divergence may be much less pronounced for more complex systems. In particular, the results of Tielens \& Hagen (1982) for abundances on evolved grain mantles appear to be adequately reproduced by the rate equation results of Hasegawa et al. (1992). Clearly, the situation must be explored in some detail.

In this paper, following Tielens, we use a simple system to explore the differences between the rate equation approach and the Monte Carlo approach to grain chemistry. We then modify the rate equations in several ways and show that with these simple modifications, the rate equations yield results that are much closer to the Monte Carlo procedure. We confirm and generalize our approach by comparing it with the results of other Monte Carlo calculations on two more complex systems. In future work, we will utilize the modified rate equation approach to redo the timedependent grain chemistry previously calculated for specific models of interstellar clouds. Our approach is semiempirical in nature and may eventually be superseded by a more detailed yet practical procedure, which currently does not exist. A semiempirical treatment of the type proposed here has ample precedence in scientific literature.

\section{A SIMPLE SYSTEM: DIATOMIC MOLECULE FORMATION}

Consider, as did Tielens, an interstellar cloud in which the gas phase consists entirely of hydrogen and oxygen atoms, with fixed concentrations $n(\mathrm{H})$ and $n(\mathrm{O})$. Hydrogen and oxygen atoms adsorb onto grains where they can associate with themselves or with each other to produce the three diatomic molecules $\mathrm{H}_{2}, \mathrm{O}_{2}$, and $\mathrm{OH}$ :

$$
\begin{gathered}
\mathrm{H}+\mathrm{H} \rightarrow \mathrm{H}_{2}, \\
\mathrm{O}+\mathrm{O} \rightarrow \mathrm{O}_{2}, \\
\mathrm{H}+\mathrm{O} \rightarrow \mathrm{OH} .
\end{gathered}
$$


These three molecules are assumed to remain on the grains and to be unreactive. Atomic hydrogen, on the other hand, can evaporate with a rather short timescale, as is thought to occur on $10 \mathrm{~K}$ grains (Tielens \& Allamandola 1987; Hasegawa et al. 1992). This simple system contains much of grain chemistry - the formation of molecular hydrogen, the formation of heavy nonhydrogenic molecules, and the formation of hydrides. It does not, as did Tielens's system, contain the formation of polyatomic molecules, a neglect that we rectify by studying more complex systems after determining how to modify the standard rate equations.

For this simple system, the rate equations governing the numbers of species $N(\mathrm{I})$ for species I on an average grain are

$$
\begin{gathered}
\frac{d N(\mathrm{H})}{d t}=k_{\mathrm{acc}}(\mathrm{H}) n(\mathrm{H})-t_{\text {evap }}^{-1}(\mathrm{H}) N(\mathrm{H}) \\
-k_{\mathrm{H}, \mathrm{H}} N(\mathrm{H}) N(\mathrm{H})-k_{\mathrm{H}, \mathrm{O}} N(\mathrm{H}) N(\mathrm{O}), \\
\frac{d N(\mathrm{O})}{d t}=k_{\mathrm{acc}}(\mathrm{O}) n(\mathrm{O})-k_{\mathrm{O}, \mathrm{O}} N(\mathrm{O}) N(\mathrm{O})-k_{\mathrm{H}, \mathrm{O}} N(\mathrm{H}) N(\mathrm{O}),
\end{gathered}
$$

$$
\begin{aligned}
\frac{d N\left(\mathrm{H}_{2}\right)}{d t} & =0.5 k_{\mathrm{H}, \mathrm{H}} N(\mathrm{H}) N(\mathrm{H}), \\
\frac{d N(\mathrm{OH})}{d t} & =k_{\mathrm{H}, \mathrm{O}} N(\mathrm{H}) N(\mathrm{O}), \\
\frac{d N\left(\mathrm{O}_{2}\right)}{d t} & =0.5 k_{\mathrm{O}, \mathrm{O}} N(\mathrm{O}) N(\mathrm{O}),
\end{aligned}
$$

where $k_{\text {acc }}(\mathrm{I})\left(\mathrm{cm}^{3} \mathrm{~s}^{-1}\right)$, the accretion rate for species $\mathrm{I}$, is given by the cross section of the grain multiplied by the average speed of species I, $n(\mathrm{I})\left(\mathrm{cm}^{-3}\right)$ is the gas-phase abundance of species $\mathrm{I}, t_{\text {evap }}^{-1}\left(\mathrm{~s}^{-1}\right)$ is the inverse of the $\mathrm{H}$ evaporation time $t_{\text {evap }}(\mathrm{H})$, and the $k_{\mathrm{I}, \mathrm{J}}$, the so-called rate coefficients, are sums of inverse diffusion times for sweeping of the entire grain $\left(t_{s}\right)$ via tunneling or thermal hopping (Hasegawa et al. 1992):

$$
k_{\mathrm{I}, \mathrm{J}}=t_{s}^{-1}(\mathrm{I})+t_{s}^{-1}(\mathrm{~J}) .
$$

The specific values for $k_{\mathrm{acc}}(\mathrm{H}), k_{\mathrm{acc}}(\mathrm{O}), t_{s}^{-1}(\mathrm{H}), t_{s}^{-1}(\mathrm{O})$, and $t_{\text {evap }}^{-1}(\mathrm{H})$ used here are listed in Table 1 and are taken from the $10 \mathrm{~K}$ results of Hasegawa et al. (1992), which are

TABLE 1

ACCRETION, SwEEPING, AND Evaporation RATES OF ACCRETING SPECIES $^{\mathrm{a}}$

\begin{tabular}{lccc}
\hline \hline Species & $\begin{array}{c}k_{\text {acc }} \\
\left(\mathrm{cm}^{3} \mathrm{~s}^{-1}\right)\end{array}$ & $\begin{array}{c}t_{s}^{-1} \\
\left(\mathrm{~s}^{-1}\right)\end{array}$ & $\begin{array}{c}t_{\text {evap }}^{-1} \\
\left(\mathrm{~s}^{-1}\right)^{\mathrm{b}}\end{array}$ \\
\hline $\mathrm{H} \ldots \ldots$. & $1.4 \times 10^{-5}$ & $5.1 \times 10^{4}$ & $1.9 \times 10^{-3}$ \\
$\mathrm{O} \ldots \ldots$. & $3.6 \times 10^{-6}$ & $4.2 \times 10^{-5}$ & $\ldots$ \\
$\mathrm{N} \ldots \ldots$. & $3.9 \times 10^{-6}$ & $4.5 \times 10^{-5}$ & $\ldots$ \\
$\mathrm{H}_{2} \ldots \ldots$ & $1.0 \times 10^{-5}$ & $3.0 \times 10^{3}$ & $6.9 \times 10^{-8}$ \\
\hline
\end{tabular}

${ }^{a}$ All quantities have been calculated for a gas and dust temperature $T=10 \mathrm{~K}$, based on the prior work of Hasegawa et al. 1992 and Hasegawa \& Herbst 1993.

${ }^{\mathrm{b}}$ Evaporation rates for $\mathrm{O}$ and $\mathrm{N}$ are $1.99 \times 10^{-23} \mathrm{~s}^{-1}$ and $2.17 \times 10^{-23} \mathrm{~s}^{-1}$, respectively; they have been assumed equal to zero here. obtained assuming classical grains with $10^{6}$ sites per grain. Nitrogen rates listed in Table 1 will be used in $\S 4$. The sweeping (migration) rate of $\mathrm{H}$ is many orders of magnitude greater than all other reactive atoms, so that surface rate coefficients for reactions involving $\mathrm{H}$ are much larger than those for reactions in which $\mathrm{H}$ does not participate.

In applications below, we sometimes refer to the accretion times $t_{\text {acc }}(\mathrm{H})$ and $t_{\text {acc }}(\mathrm{O})$; these quantities are the time intervals between successive $\mathrm{H}$ and $\mathrm{O}$ species landing on a particular grain. Mathematically,

$$
t_{\mathrm{acc}}^{-1}(\mathrm{H})=k_{\mathrm{acc}}(\mathrm{H}) n(\mathrm{H}) \text {, }
$$

with an analogous equation for $\mathrm{O}$.

For comparison with the results obtained via numerical integration of the rate equations, we have constructed a Monte Carlo model in which $\mathrm{H}$ and $\mathrm{O}$ atoms randomly land on an individual grain according to the grain collision frequency. If an $\mathrm{H}$ atom lands, it will either react or evaporate, while if an $\mathrm{O}$ atom lands it will react with the previous adsorbate (if it is still present) or wait until the next adsorbate lands. This simple set of rules works reasonably well for this simple system. Indeed, for very slow and very rapid rates of accretion with respect to the $\mathrm{H}$ evaporation rate, the Monte Carlo results reduce to a simple analytical model in which the production of species on grain surfaces is simply related to the atomic fractional abundances in the gas and the velocities of the gas-phase species. There is a slight complication when $n(\mathrm{H})>n(\mathrm{O})$ and $n(\mathrm{O})$ is sufficiently large because there is the possibility that an $\mathrm{H}$ atom landing subsequently to two $\mathrm{O}$ atoms will, because of its rapid migration, react first with one of the $\mathrm{O}$ atoms. However, the probability of two $\mathrm{O}$ atoms landing in a row is small, since we do not consider atomic oxygen gas-phase concentrations large enough for this complication to be important.

The results of the model as solved by the grain rate equations are shown in Figure 1 (top panel) for models in which $n(\mathrm{O})=1 \mathrm{~cm}^{-3}$ and $n(\mathrm{H})$ varies from 0.1 to $10,000 \mathrm{~cm}^{-3}$. These models represent clouds at various ages; detailed gasphase models show that the $\mathrm{O}$ atom abundance is close to what is assumed here at all times, whereas the $\mathrm{H}$ atom abundance decreases from its initial value, at which time a significant portion of the $\mathrm{H}$ elemental abundance is in atomic form, to a final value in which most of the $\mathrm{H}$ elemental abundance is molecular. Figure 1 (top panel) contains a plot of the fractional abundances on grain surfaces (labeled mole fractions $X$ ) of the three diatomic molecules produced as functions of $n(\mathrm{H})$ once these fractional abundances reach constant values; the fractional abundances of the atoms are negligible. In Figure 1 (bottom panel), we present the analogous results based on the Monte Carlo calculation.

One can see two "phase transitions" in the plots as a function of increasing $n(\mathrm{H})$, the first occurring when the dominant grain molecule switches from $\mathrm{O}_{2}$ to $\mathrm{OH}$, the second when the dominant grain molecule switches from $\mathrm{OH}$ to $\mathrm{H}_{2}$. The results obtained with the grain-rate equations show the first phase transition to occur at an $n(\mathrm{H})$ value lower than the minimum plotted in the figure, while the second phase transition $\left(\mathrm{OH} \rightarrow \mathrm{H}_{2}\right)$ occurs at $n(\mathrm{H}) \approx 1$ $\mathrm{cm}^{-3}$. The analogous results from the Monte Carlo calculation show that the phase transitions occur at far larger values of $n(\mathrm{H})$; for $\mathrm{O}_{2} \rightarrow \mathrm{OH}$, the transition occurs at $n(\mathrm{H}) \approx 0.2-0.3 \mathrm{~cm}^{-3}$ whereas for $\mathrm{OH} \rightarrow \mathrm{H}_{2}$, the transition occurs at $n(\mathrm{H}) \approx 30 \mathrm{~cm}^{-3}$. Clearly, as Tielens noted, 


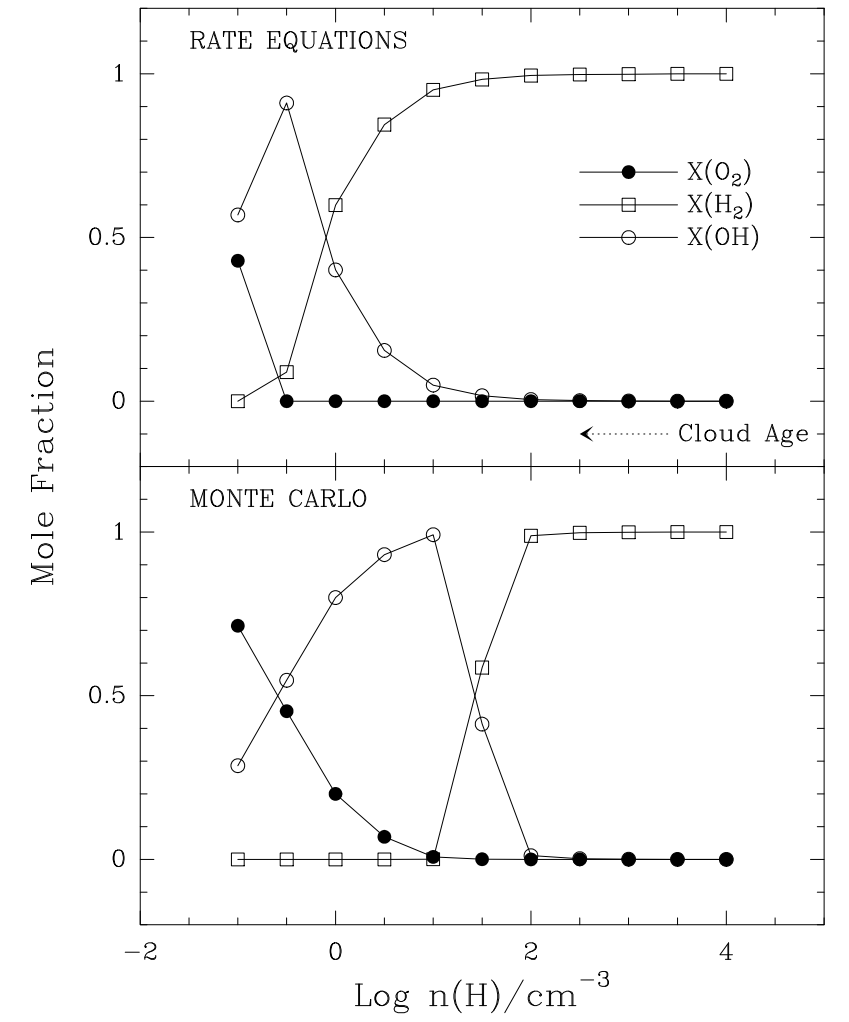

Fig. 1.-Fractional abundances relative to the total number of surface species (mole fraction) of surface $\mathrm{O}_{2}, \mathrm{H}_{2}$, and $\mathrm{OH}$ vs. gas-phase number density of atomic hydrogen, $n(\mathrm{H})$, from (top) rate equation calculations and (bottom) Monte Carlo calculations. The gas-phase number density of oxgyen atoms, $n(\mathrm{O})$, is fixed at a value of $1 \mathrm{~cm}^{-3}$, and the temperature is 10 $\mathrm{K}$. Note the large discrepancy between the two methods for $n(\mathrm{H})<100$ $\mathrm{cm}^{-3}$. Low values of $n(\mathrm{H})$ are representative of evolved dark clouds, where hydrogen is mostly in molecular form.

there is a large discrepancy between the two approaches; the rate equation method overestimates the reaction efficiency of $\mathrm{H}$ atoms in forming $\mathrm{OH}$ and $\mathrm{H}_{2}$ molecules by a large factor.

\section{MODIFICATIONS OF RATE EQUATIONS}

In this section, we discuss some modifications to the rate equations that bring the solutions into near agreement with those of the Monte Carlo approach. These modifications are certainly neither unique nor derivable; rather they are motivated by a belated appreciation for the shortcomings of the rate equation approach. There appear to be two serious problems with the rate equations for the simple system considered. The first concerns the reaction efficiency of atomic $\mathrm{H}$ in finding reactant partners via random motions on the grain particles. Consider an $\mathrm{H}$ atom landing on a particular dust particle at a given time on which there resides another reactive species labeled $\mathrm{X}$ (here $\mathrm{X}=\mathrm{H}$ or $\mathrm{O}$ ). The fractional probability of reaction between $\mathrm{H}$ and $\mathrm{X}$ in a time period $\Delta t$ is simply $\approx t_{s}^{-1}(\mathrm{H}) \Delta t$, where we have ignored the sweeping rate of $X$ and the possibility that the reaction efficiency $\kappa(\mathrm{H}, \mathrm{X})$ is less than unity due to activation energy. The probability of reaction should not exceed unity within a physically meaningful time period, defined by the smaller of (1) the accretion time for $\mathrm{H}$ and (2) the evaporation time for $\mathrm{H}$. If the reaction probability does exceed unity, which is typically the case because of the rapid sweeping rate of $\mathrm{H}$, then it is unphysically large and must be reduced to at most unity. This can be accomplished by reducing the sweeping rate $t_{s}^{-1}(\mathrm{H})$ to the greater of the evaporation rate and accretion rate. One might ask why not just reduce the sweeping rate to the accretion rate? The reason is that in the limit when the evaporation rate exceeds the accretion rate, reducing the sweeping rate to the slower accretion rate means that hydrogenation will not be $100 \%$ efficient when an $\mathrm{H}$ lands upon a grain with one atom $\mathrm{X}$ already present, which is unphysical. In this "evaporative" limit, the $\mathrm{H}$ atom evaporation time can then be regarded as the quantum of time, since it is the shortest time interval on which events can happen.

We have so far been discussing the situation in which there is exactly one atom $X$ on the grain. If $X$ is reactive, $N(\mathrm{X})$ will generally be less than unity, but if $\mathrm{X}$ is unreactive, $N(\mathrm{X})$ may become quite large. The treatment of slow H-X reactions requires that the relative rates of fast and slow reactions of $\mathrm{H}$ atoms remain the same and that the overall reaction probability per $\mathrm{H}$ atom be less than or equal to unity. Since H-X reactions with activation energy are not included in our simple systems, we defer their discussion until $\S 6$. For a heavy species $\mathrm{X}$, such as atomic $\mathrm{O}$ landing on a grain, the sweeping rate is normally sufficiently small compared with the greater of the $\mathrm{H}$ atom evaporation and accretion rates that no correction is needed.

If we define $x$ by the equation

$$
x=t_{\text {evap }}^{-1}(\mathrm{H}) / t_{\text {acc }}^{-1}(\mathrm{H}),
$$

then, for $x<1$, we have the following equations for the modified rate coefficients for our simple system:

$$
\begin{aligned}
& k_{\mathrm{H}, \mathrm{H}}=t_{\mathrm{acc}}^{-1}(\mathrm{H})+t_{\mathrm{acc}}^{-1}(\mathrm{H}), \\
& k_{\mathrm{H}, \mathrm{O}}=t_{\mathrm{acc}}^{-1}(\mathrm{H})+t_{s}^{-1}(\mathrm{O}), \\
& k_{\mathrm{O}, \mathrm{O}}=t_{s}^{-1}(\mathrm{O})+t_{s}^{-1}(\mathrm{O}),
\end{aligned}
$$

while, for $x \geq 1$, we have

$$
\begin{gathered}
k_{\mathrm{H}, \mathrm{H}}=t_{\text {evap }}^{-1}(\mathrm{H})+t_{\text {evap }}^{-1}(\mathrm{H}), \\
k_{\mathrm{H}, \mathrm{O}}=t_{\text {evap }}^{-1}(\mathrm{H})+t_{s}^{-1}(\mathrm{O}), \\
k_{\mathrm{O}, \mathrm{O}}=t_{s}^{-1}(\mathrm{O})+t_{s}^{-1}(\mathrm{O}) .
\end{gathered}
$$

Using the results in Table 1 , it can be seen that $x=1$ at $n(\mathrm{H}) \approx 100 \mathrm{~cm}^{-3}$, so that for current dense cloud conditions, in which most gas-phase hydrogen is in molecular form, $x \gg 1$.

The results of the rate equation model with the above modified rate coefficients are shown in Figure $2 a$, where it can be seen that there is still a serious disagreement with the results of the Monte Carlo calculation. In particular, the $\mathrm{OH} \rightarrow \mathrm{H}_{2}$ transition occurs at a hydrogen atom concentration a factor of 4 too low, indicating that the production of molecular hydrogen is still too efficient. In addition, the $\mathrm{O}_{2} \rightarrow \mathrm{OH}$ transition now occurs at too high a gas-phase $\mathrm{H}$ concentration by a factor of 2 .

The overproduction of molecular hydrogen at low atomic hydrogen gas densities occurs due to the second major problem involving rate equations. Specifically, it occurs because we have not adequately taken into account the evaporation of atomic hydrogen from a grain surface before another atom can land on the same grain. If we neglect the presence of atomic $\mathrm{O}$, the rate of molecular hydrogen formation on a grain when evaporation is dominant $(x>1)$ is given by the expression

$$
d N\left(\mathrm{H}_{2}\right) / d t \approx t_{\mathrm{acc}}^{-1}(\mathrm{H}) \exp (-x),
$$




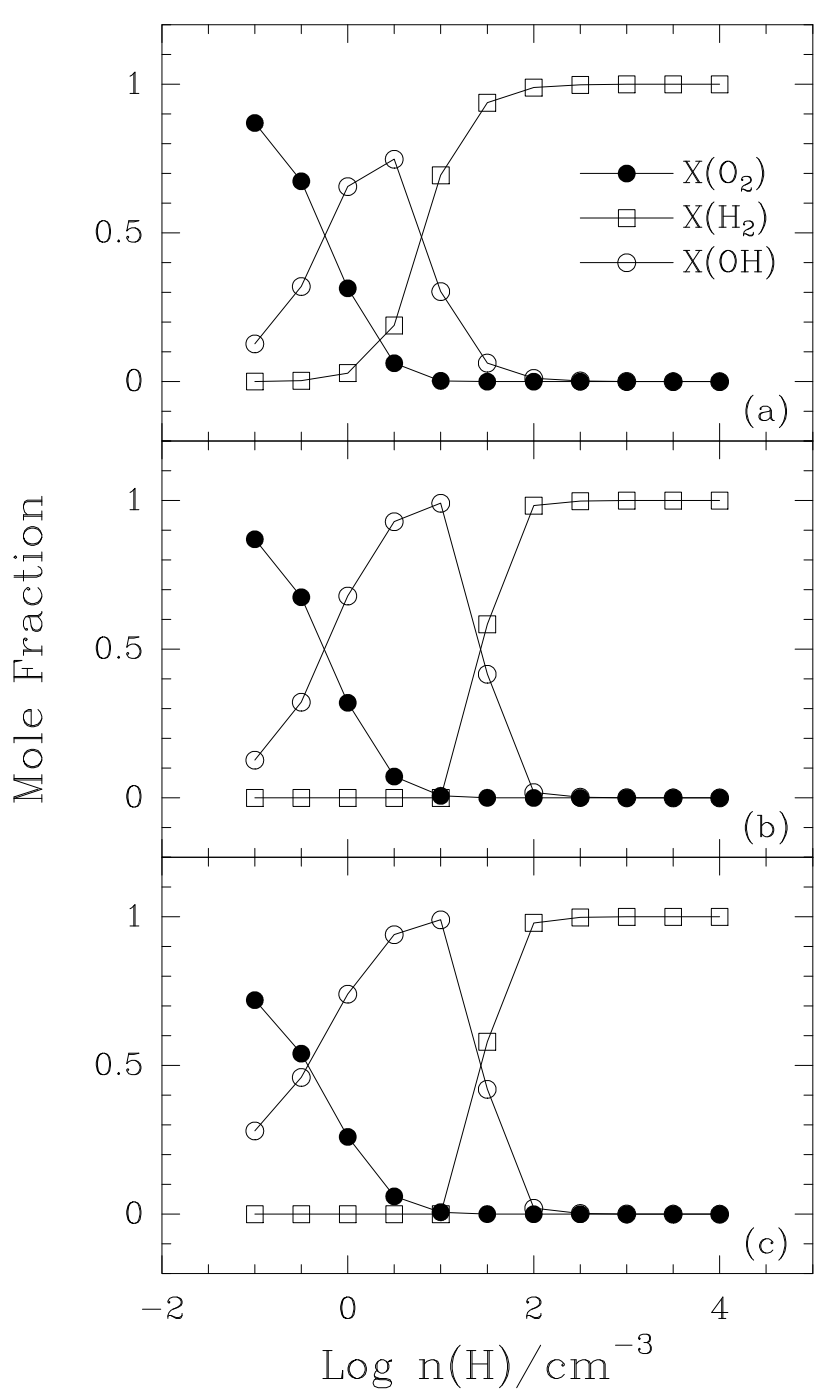

Fig. 2.-Same plot as in Fig. 1, but here showing the continual improvement of the rate equation method after the inclusion of the three modifications discussed in the text: $(a)$ reduction in the sweeping rate of atomic hydrogen, $(b)$ inclusion of evaporation of $\mathrm{H}$ in the expression for the production of $\mathrm{H}_{2}$, and $(c)$ modification of the rate equation for the production of $\mathrm{OH}$. Note the similarity between this figure, where all modifications are included, and Fig. 1 (bottom), which shows the Monte Carlo results.

the physical meaning of which is that after one accretion time, the probability of an $\mathrm{H}$ atom remaining on the grain to react with the next $\mathrm{H}$ that lands is $\exp (-x)$. This result is not obtained by approximate solution of the kinetic equation for $\mathrm{H}_{2}$ formation in the evaporative limit $(x>1)$, unless the rate coefficient $k_{\mathrm{H}, \mathrm{H}}$ is modified to its final form:

$$
k_{\mathrm{H}, \mathrm{H}}=\left[t_{\text {evap }}^{-1}(\mathrm{H})+t_{\text {evap }}^{-1}(\mathrm{H})\right] x \exp (-x) .
$$

In Figure $2 b$, we plot the results of the rate equation approach with the final correction for $k_{\mathrm{H}, \mathrm{H}}$ and find apparent agreement with the Monte Carlo approach for the $\mathrm{OH} \rightarrow \mathrm{H}_{2}$ transition.

Despite these two major corrections, there is still a relatively small problem remaining with the results of the rate equation model: an overproduction of $\mathrm{O}_{2}$ and an underproduction of $\mathrm{OH}$ at low $\mathrm{H}$ gas densities. This remaining problem occurs under conditions in which the $\mathrm{O}$ atom accretion rate is appreciable, and suggests that we have overcompensated in reducing the $\mathrm{H}$ reaction efficiency. A very simple remedy is to reduce the rate of $\mathrm{H}$-atom sweeping not to $t_{\text {evap }}^{-1}(\mathrm{H})$ but to a somewhat larger value such as twice this rate. This modification works reasonably well but, overall, is not as good as a specific empirical correction factor to enhance $k_{\mathrm{H}, \mathrm{O}}$, which we have adopted. With this correction for $x \geq 1$, the rate coefficient for the formation of $\mathrm{OH}$ becomes

$$
\begin{aligned}
k_{\mathrm{H}, \mathrm{O}}^{\prime} & =k_{\mathrm{H}, \mathrm{O}}+k_{\mathrm{H}, \mathrm{O}} \frac{t_{\mathrm{acc}}^{-1}(\mathrm{O})}{t_{\mathrm{acc}}^{-1}(\mathrm{H})} \\
& =\left[t_{\mathrm{evap}}^{-1}(\mathrm{H})+t_{s}^{-1}(\mathrm{O})\right]\left[1+\frac{t_{\mathrm{acc}}^{-1}(\mathrm{O})}{t_{\mathrm{acc}}^{-1}(\mathrm{H})}\right] .
\end{aligned}
$$

The corrected rate $k_{\mathrm{H}, \mathrm{O}}^{\prime}>k_{\mathrm{H}, \mathrm{O}}$ when the probability for $\mathrm{O}$ to land first is significantly greater than zero $\left(k_{\mathrm{H}, \mathrm{o}}^{\prime} / k_{\mathrm{H}, \mathrm{O}}=\right.$ $1.25-3.5$ if $n(\mathrm{H})=1.0-0.1 \mathrm{~cm}^{-3}$, while $\left.n(\mathrm{O})=1.0 \mathrm{~cm}^{-3}\right)$. When $t_{\mathrm{acc}}^{-1}(\mathrm{H}) \gg t_{\mathrm{acc}}^{-1}(\mathrm{O}), k_{\mathrm{H}, \mathrm{O}}^{\prime}$ simply reduces to $k_{\mathrm{H}, \mathrm{O}}$ and equation (20) reduces to equation (16). The use of equation (20) instead of equation (16) leads to a general agreement with the Monte Carlo approach, shown in a comparison between the results in Figures 1 (bottom panel) and 2c. Still, since our last correction is empirical, its importance in detailed model calculations (Shalabiea, Caselli, \& Herbst 1998) will have to be carefully monitored.

\section{PRODUCTION OF $\mathrm{H}_{2} \mathrm{O}$ AND $\mathrm{NH}_{3}$}

A more detailed comparison among the various approaches can be obtained by comparing yearly production rates of molecules on the grain surface rather than by comparing fractional abundances of the dominant species. For this purpose and to extend our analysis to polyatomic molecules, the simple chemical system described in $\S 2$ has been extended to include the additional accreting atom $\mathrm{N}$ (see Table 1) and the formation of the additional surface species $\mathrm{NH}, \mathrm{NO}, \mathrm{N}_{2}, \mathrm{NH}_{2}, \mathrm{H}_{2} \mathrm{O}$, and $\mathrm{NH}_{3}$. The reactions added to the model include

$$
\begin{aligned}
\mathrm{N}+\mathrm{H} & \rightarrow \mathrm{NH}, \\
\mathrm{N}+\mathrm{N} & \rightarrow \mathrm{N}_{2}, \\
\mathrm{~N}+\mathrm{O} & \rightarrow \mathrm{NO}, \\
\mathrm{H}+\mathrm{NH} & \rightarrow \mathrm{NH}_{2}, \\
\mathrm{H}+\mathrm{OH} & \rightarrow \mathrm{H}_{2} \mathrm{O}, \\
\mathrm{H}+\mathrm{NH}_{2} & \rightarrow \mathrm{NH}_{3} .
\end{aligned}
$$

As before, evaporation rates are assumed equal to zero, except for atomic hydrogen.

We focus on the $x \geq 1$ (evaporative) case, where the results of the unmodified rate equations deviate most from the Monte Carlo method (see Fig. 1). In addition to the fully modified rate coefficients developed in the last section, the other rate coefficient expressions used are

$$
\begin{gathered}
k_{\mathrm{H}, \mathrm{OH}}=t_{\text {evap }}^{-1}(\mathrm{H})+t_{s}^{-1}(\mathrm{OH}), \\
k_{\mathrm{H}, \mathrm{N}}=\left[t_{\text {evap }}^{-1}(\mathrm{H})+t_{s}^{-1}(\mathrm{~N})\right]\left[1+t_{\mathrm{acc}}^{-1}(\mathrm{~N}) / t_{\mathrm{acc}}^{-1}(\mathrm{H})\right], \\
k_{\mathrm{N}, \mathrm{N}}=t_{s}^{-1}(\mathrm{~N})+t_{s}^{-1}(\mathrm{~N}), \\
k_{\mathrm{N}, \mathrm{O}}=t_{s}^{-1}(\mathrm{~N})+t_{s}^{-1}(\mathrm{O}), \\
k_{\mathrm{H}, \mathrm{NH}}=t_{\text {evap }}^{-1}(\mathrm{H})+t_{\text {evap }}^{-1}(\mathrm{H}), \\
k_{\mathrm{H}, \mathrm{NH}_{2}}=t_{\text {evap }}^{-1}(\mathrm{H})+t_{s}^{-1}\left(\mathrm{NH}_{2}\right),
\end{gathered}
$$


with $10 \mathrm{~K}$ sweeping rates taken/derived from Hasegawa et al. (1992). Since the derived sweeping rate for $\mathrm{NH}, t_{s}^{-1}(\mathrm{NH})$, exceeds the evaporation rate for $\mathrm{H}$, a correction analogous to the one applied to atomic hydrogen has been made to avoid unphysically large reaction probabilities (cf. § 3).

Results and comparison with a Monte Carlo method are shown in Figure 3 for $n(\mathrm{O})=1 \mathrm{~cm}^{-3}, n(\mathrm{~N})=0.12 \mathrm{~cm}^{-3}$, and $n(\mathrm{H})$ varying from 0.1 to $100 \mathrm{~cm}^{-3}$, where the yearly production $N(\mathrm{I})$ of assorted surface species is plotted versus $n(\mathrm{H})$. The biggest discrepancies between the two methods are about a factor of 2 , confirming the validity of the modified rate equation approach as an adequate substitute for the Monte Carlo calculation. The Monte Carlo program used here, based on the work of Tielens \& Hagen (1982), is more general than that used for the simple diatomic system and includes the use of rate coefficients to determine relative reaction probabilities for a species landing on a grain if more than one other type of species is already present.

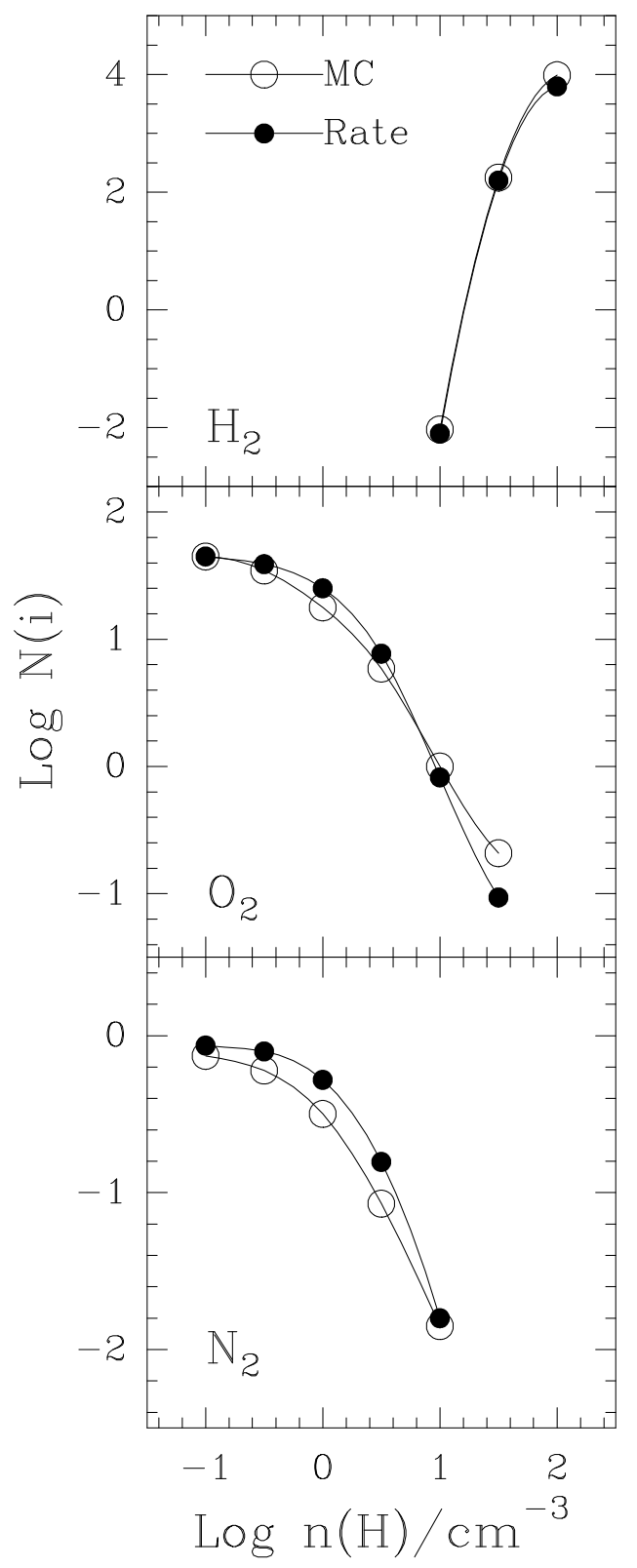

\section{HYDROGENATION VIA $\mathrm{H}_{2}$ REACTIONS: SURFACE REACTIONS WITH ACTIVATION ENERGY BARRIERS}

Hydrogenation by grain reactions involving atomic hydrogen typically occurs without activation energy and involves a competition as to whether an accreting hydrogen will find a reactive species on the grain or evaporate. This mechanism is clearly in the so-called "accretion limit" and, as we have seen, requires modification to the rate equations. Hydrogenation via reaction with molecular hydrogen, if it occurs competitively on grain surfaces, is a completely different matter. Molecular hydrogen is a rather nonreactive species, and reactions are likely to occur inefficiently due to activation energy barriers. The surface population of $\mathrm{H}_{2}$ is likely to be quite large and in the outermost mantle layers, however, especially in dense clouds, where the number density of gas-phase molecular hydrogen $n\left(\mathrm{H}_{2}\right) \sim 10^{3}-10^{4}$ $\mathrm{cm}^{-3}$, while $n(\mathrm{H}) \sim 1 \mathrm{~cm}^{-3}$ (the case $x \geq 1$ applies, see $\S 3$ ),

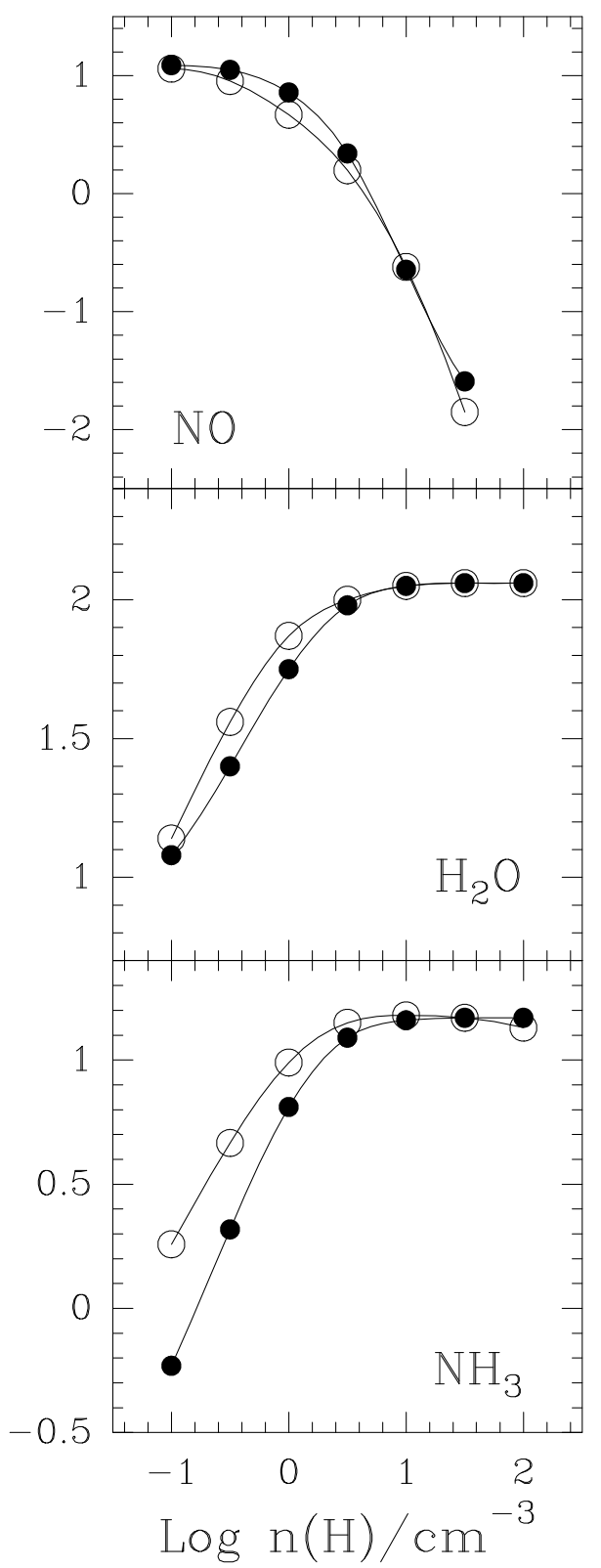

FIG. 3.-Yearly production of surface species $N(\mathrm{i})$ vs. $n(\mathrm{H})$ calculated with the Monte Carlo method (open circles) and the modified rate equations ( filled circles). Fixed parameters in the models are $n(\mathrm{O})=1 \mathrm{~cm}^{-3}, n(\mathrm{~N})=1.2 \times 10^{-1} \mathrm{~cm}^{-3}$, and $T=10 \mathrm{~K}$. The biggest differences between the two methods are around a factor of 2 . Slow convergence in the Monte Carlo method rules out the accurate calculation of the abundances of trace species. 

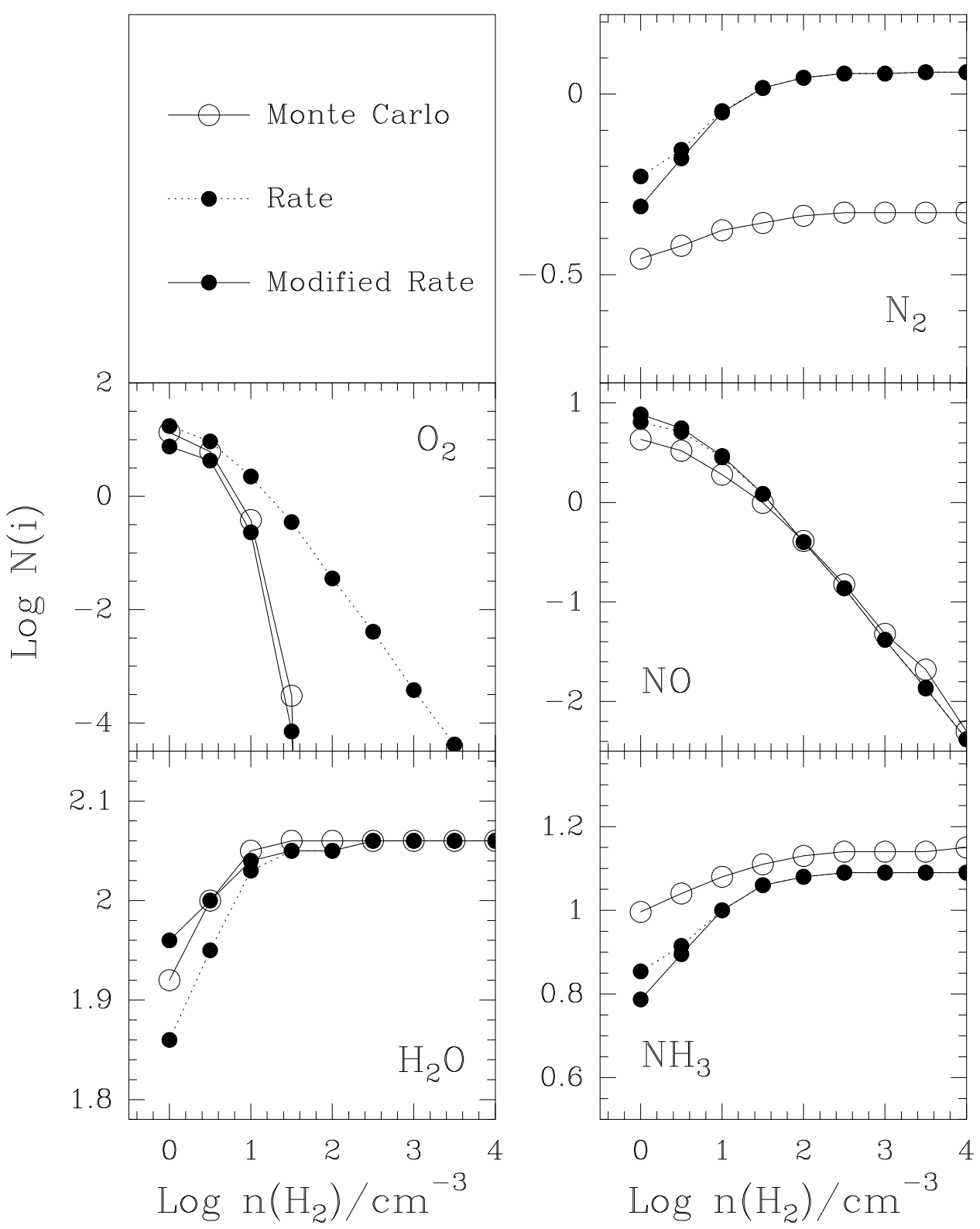

FIG. 4.-Yearly production of surface species $\mathrm{N}(\mathrm{i})$ vs. $n\left(\mathrm{H}_{2}\right)$ calculated with the Monte Carlo method (open circles) and rate equations (filled circles). Dotted lines connect points calculated with the rate equation approach without modification of the $\mathrm{O}_{2}$ formation rate but with all earlier modifications, while solid lines connect points calculated with the rate equation approach with the $\mathrm{O}_{2}$ rate suitably modified. Fixed parameters in the models are $n(\mathrm{O})=1 \mathrm{~cm}{ }^{-3}$, $n(\mathrm{~N})=1.2 \times 10^{-1} \mathrm{~cm}^{-3}$, and $T=10 \mathrm{~K}$. The surface abundance of $\mathrm{H}_{2}$ is kept at the steady state value (see text). The major difference between the Monte Carlo and unmodified rate results concerns $\mathrm{O}_{2}$; modification of the rate equation for $\mathrm{O}_{2}$ formation removes the discrepancy.

so that the overall possibility of hydrogenation of an accreting species by reaction with $\mathrm{H}_{2}$ may be competitive. In particular, if we include the evaporation rate of $\mathrm{H}_{2}$ (see Table 1) in our previous model and assume that $n\left(\mathbf{H}_{2}\right)=$ $10^{3} \mathrm{~cm}^{-3}, \mathrm{~N}\left(\mathrm{H}_{2}\right) \approx 4 \times 10^{4}$ after only $\sim 0.2 \mathrm{yr}$ and continues to increase rapidly until a steady state population of $1.48 \times 10^{5} \mathrm{H}_{2}$ molecules per grain is reached (after $\sim 2.5$ yr). More generally, the steady-state surface population of $\mathrm{H}_{2}$ is $148 \times n\left(\mathrm{H}_{2}\right)$.

The rate coefficient for the surface reaction between $\mathrm{X}$ and $\mathrm{H}_{2}$ is given by the expression

$$
k_{\mathrm{X}, \mathrm{H}_{2}}=\kappa\left[t_{s}^{-1}(\mathrm{X})+t_{s}^{-1}\left(\mathrm{H}_{2}\right)\right],
$$

where $\kappa$ is the probability for the reaction to occur upon an encounter. This factor is obtained by using a simple tunnel- ing approximation (Tielens \& Hagen 1982; Hasegawa et al. 1992). Such reactions were found to be very important in gas-grain models where the surface chemistry was treated by unmodified rate equations if the activation energy is in the vicinity of $2100 \mathrm{~K}$ (Hasegawa \& Herbst 1993). Since the rate equation approach should be plausible for large numbers of reactant species on a grain and since the mechanism for reaction is not accretion of an $\mathrm{H}_{2}$ followed by a competition between desorption and reaction, the reaction rate coefficient $k_{\mathrm{X}, \mathrm{H}_{2}}$ is unlikely to need modification in a rate treatment. (Of course, if the surface $\mathrm{H}_{2}$ abundance becomes sufficiently large, then the sweeping LangmuirHinshelwood mechanism gives way to an Eley-Rideal mechanism (Somorjai 1994), in which sweeping rates must be replaced by a vibrational frequency between $\mathrm{O}$ and $\mathrm{H}_{2}$.) 
What may be necessary is a modification to the rate coefficients for formation of species XY when either one or both of the species can also react competitively on the surface with $\mathrm{H}_{2}$.

Consider a heavy reactive species $X$ landing on a grain rich in $\mathrm{H}_{2}$ molecules. Before the accretion of a subsequent species that might react rapidly with $\mathrm{X}$, there is a possibility of reaction between $\mathrm{X}$ and molecular hydrogen. If we let $x^{\prime}$ be the ratio of the reaction rate between $\mathrm{X}$ and surface $\mathrm{H}_{2}$ to the total accretion rate $t_{\mathrm{acc}}^{-1}$ of reactive species; viz.,

$$
x^{\prime}=k_{\mathrm{X}, \mathrm{H}_{2}} N\left(\mathrm{H}_{2}\right) / t_{\mathrm{acc}}^{-1},
$$

then the probability that $X$ will be unreacted when the next reactive species accretes is given by the simple formula exp $\left(-x^{\prime}\right)$ in the limit $x^{\prime} \gg 1$. The exponential probability is built into our Monte Carlo analysis but not into the rate analysis. The simplest modification to use in the rate equation approach is obtained via the same logic that led to the correction for $\mathrm{H}_{2}$ formation; namely, the multiplication of the rate coefficient for XY formation by $x^{\prime} \exp \left(-x^{\prime}\right)$, for $x^{\prime}>1$, where $x^{\prime}$ is the lesser of the two ratios defined for species $\mathrm{X}$ and $\mathrm{Y}$.

To analyze the possible importance of hydrogenation by $\mathrm{H}_{2}$, we have added the "fictitious" reaction

$$
\mathrm{O}+\mathrm{H}_{2} \rightarrow \mathrm{H}_{2} \mathrm{O}
$$

to the reaction network described in $\S 4$. The additional reaction has been assigned an activation energy of $2100 \mathrm{~K}$ (far smaller than its actual value) that gives it a reaction rate coefficient of $4.5 \times 10^{-8} \mathrm{~s}^{-1}$ at $10 \mathrm{~K}$ with the standard tunneling assumptions, which are highly uncertain to say the least. We have run a variety of model calculations, with fixed gas-phase abundances for $\mathrm{H}, \mathrm{O}$, and $\mathrm{N}$ of $n(\mathrm{H})=n(\mathrm{O})=1 \mathrm{~cm}^{-3}$, and $n(\mathrm{~N})=0.12 \mathrm{~cm}^{-3}$, respectively, while the gas-phase molecular hydrogen abundance $n\left(\mathrm{H}_{2}\right)$ is varied between 1 and $10^{4} \mathrm{~cm}^{-3}$, and the surface molecular hydrogen abundance is fixed at the steady state value $N\left(\mathrm{H}_{2}\right)=148 n\left(\mathrm{H}_{2}\right)$. The fixed value for surface $\mathrm{H}_{2}$ is used to facilitate comparison with a Monte Carlo calculation. For this purpose, the general Monte Carlo program based on the work of Tielens \& Hagen (1982) that we have written must itself be modified, since the dominant accretor is not a reactive species.

Results for the surface population yearly production rates are shown in Figure 4 for the species $\mathrm{N}_{2}, \mathrm{O}_{2}, \mathrm{NO}$, $\mathrm{H}_{2} \mathrm{O}$, and $\mathrm{NH}_{3}$. In this figure, we plot the Monte Carlo results, the rate results with no additional modifications, and the rate results with the additional modification for the rate of $\mathrm{O}_{2}$ formation. It can immediately be seen that the agreement between the Monte Carlo results and the modified rate equation results is uniformly good to within a factor of a few. In both calculations, surface $\mathrm{O}_{2}$ vanishes beyond a certain $\mathrm{H}_{2}$ density because the reaction of accreting oxygen atoms with surface $\mathrm{H}_{2}$ occurs with near $100 \%$ efficiency before another $\mathrm{O}$ atom can land on an individual grain. Without the modification to the rate equations, the surface abundance of $\mathrm{O}_{2}$ still becomes quite low as the $\mathrm{H}_{2}$ density increases, but the value is much higher than the correct one. This type of result was obtained by Hasegawa \& Herbst (1993).

\section{CONCLUSIONS}

We have confirmed Tielens's calculation that for a simple system of grain equations, the rate equation approach com- monly used by ourselves and other scientists does not reproduce the results of Monte Carlo calculations, which take into account the discrete nature of grains. Several simple modifications to the rate coefficients used in the rate equations, however, have the result of rendering the rate approach a useful substitute for the Monte Carlo approach. The modifications are hardly unique and are designed for simplicity of use in time-dependent gas-grain models, for which use of the Monte Carlo approach would be laborious at best. The modifications are designed for $10 \mathrm{~K}$ grains. For grain temperatures greater than $10 \mathrm{~K}$, the Monte Carlo technique for our simplest model shows little if any temperature dependence until the evaporation rate for $\mathrm{H}$ equals the sweeping rate, which occurs at 17-20 K. At greater temperatures, $\mathrm{H}$ is unreactive on surfaces because its evaporation rate is so large. To use the modified rate approach at temperatures up to $17 \mathrm{~K}$ in the evaporative limit (which is essentially the only limit for $T>10 \mathrm{~K}$ ), calculations on the simplest model suggest that it is best to leave the $10 \mathrm{~K}$ evaporation rate for $\mathrm{H}$ unchanged except for $k_{\mathrm{H}, \mathrm{H}}$ and to make the necessary changes in the other rates (Hasegawa et al. 1992). The reason for this approach is that our empirical correction, which depends heavily on the evaporation rate of $\mathrm{H}$, has been adjusted at $10 \mathrm{~K}$. With this simple procedure, we find good agreement with the Monte Carlo technique at temperatures up to $17 \mathrm{~K}$ for the $\mathrm{O}, \mathrm{H}$ system.

The $10 \mathrm{~K}$ modifications are summarized below:

1. If $x \equiv t_{\text {evap }}^{-1}(\mathrm{H}) / t_{\mathrm{acc}}^{-1}(\mathrm{H}) \geq 1$, the sweeping rate $t_{s}^{-1}(\mathrm{H})$ is reduced to the evaporation rate $t_{\text {evap }}^{-1}(\mathrm{H})$, and the rate coefficient for the surface formation of $\mathrm{H}_{2}$ is given by equation (19). For reactions without activation energy between atomic hydrogen and a heavy species $X$, rate coefficient expressions have the form

$$
k_{\mathrm{H}, \mathrm{X}}=\left[t_{\mathrm{evap}}^{-1}(\mathrm{H})+t_{s}^{-1}(\mathrm{X})\right]\left[1+\frac{t_{\mathrm{acc}}^{-1}(\mathrm{X})}{t_{\mathrm{acc}}^{-1}(\mathrm{H})}\right],
$$

while for all the other surface reactions involving heavy species, rate coefficients are unchanged. If, however, a particular heavy species (e.g., $\mathrm{NH}$ ) is calculated to have a sweeping rate faster than the evaporation rate of $\mathrm{H}$, its sweeping rate must be reduced to the evaporation rate. If, in addition, the heavy species can react appreciably with $\mathrm{H}_{2}$, its rate coefficients for reaction with other heavy species may need to be modified further (see stipulation 3 below).

2. If $x<1$, the sweeping rate $t_{s}^{-1}(\mathrm{H})$ is reduced to the accretion rate $t_{\mathrm{acc}}^{-1}(\mathrm{H})$. Formation of $\mathrm{H}_{2}$ proceeds with a rate given by equation (12). For all the other surface reactive species, sweeping rates are not modified (as long as $\left.t_{s}^{-1}[\mathrm{X}]<t_{\mathrm{acc}}^{-1}[\mathrm{H}]\right)$, and rate coefficients have the form

$$
k_{\mathrm{H}, \mathrm{X}}=t_{\mathrm{acc}}^{-1}(\mathrm{H})+t_{s}^{-1}(\mathrm{X})
$$

for reactions involving $\mathrm{H}$, and (unless stipulation 3 comes into play)

$$
k_{\mathrm{X}, \mathrm{Y}}=t_{s}^{-1}(\mathrm{X})+t_{s}^{-1}(\mathrm{Y})
$$

for surface reactions involving heavy species $\mathrm{X}$ and $\mathrm{Y}$. If $t_{s}^{-1}(\mathrm{X})>t_{\mathrm{acc}}^{-1}(\mathrm{H})$, the sweeping rate of species $\mathrm{X}$ must be reduced to the accretion rate of $H$.

3. If there is a large surface population of $\mathrm{H}_{2}$ (which occurs under mature dense cloud conditions), it may be necessary to modify not the rate of reaction between a surface species and $\mathrm{H}_{2}$ but the rate of reaction between two 
heavy surface species, if either of them can react with $\mathrm{H}_{2}$, albeit slowly. In particular, the reaction rate coefficient between two species $\mathrm{X}$ and $\mathrm{Y}$ must be reduced by the factor $x^{\prime} \exp \left(-x^{\prime}\right)$, if $x^{\prime} \geq 1$, where $x^{\prime}$ is the lesser of the two ratios (for $\mathrm{X}$ and for $\mathrm{Y}$, respectively) of the reaction rate with $\mathrm{H}_{2}$ divided by the total accretion rate for reactive species. The rate coefficient then takes the form

$$
k_{\mathrm{X}, \mathrm{Y}}^{\prime}=k_{\mathrm{X}, \mathrm{Y}} x^{\prime} \exp \left(-x^{\prime}\right) \text {. }
$$

If $x^{\prime}<1$, no correction is needed.

4. For more complex grain chemical networks than those considered here, there is a possibility that when an atomic hydrogen lands on a grain, it will be able to react with a choice of reactant partners, some of which it reacts with rapidly and others of which it reacts with slowly due to activation energy barriers. Consider, for example, a hydrogen atom accreting in the $x>1$ regime onto a grain with a large population $N(\mathrm{Y})$ of species $\mathrm{Y}$. The hydrogen atom can react rapidly with species $\mathrm{X}$ and slowly with species $\mathrm{Y}$. In this instance, we would reduce both rate coefficients using rules 1 and 2 so that the relative rates for the $\mathrm{H}-\mathrm{X}$ and $\mathrm{H}-\mathrm{Y}$ reactions remained the same. Thus, the rate coefficient of the $\mathrm{H}-\mathrm{X}$ reaction would be given by equation (35), while the rate coefficient of the H-Y reaction would require an additional factor $\kappa$ for the activation energy barrier (Hasegawa et al. 1992). If, despite this reduction, the overall probability of reaction for $\mathrm{H}$ exceeded unity, the rate coefficients would have to be normalized.

With the assorted modifications discussed here, the convenient rate equation approach to grain surface chemistry reproduces the results of Monte Carlo methods for some simple systems. Our next plan is to use the modified rate equations in time-dependent complex gas-grain chemical models of dense interstellar clouds. Here we expect that the modifications will not lead to effects as great as in the simple systems reported in this paper, because of the large numbers of competitive pathways. If the effects are dramatic, however, it may be necessary to compare calculated surface abundances with those derived from a Monte Carlo method. We will be particularly careful to monitor any effect caused by the empirical correction discused in $\S 3$ since this has little physical basis. Preliminary calculations with our modified rates using the earlier gas-grain models of Hasegawa \& Herbst (1993), which represent dark interstellar clouds at $10 \mathrm{~K}$, indicate that all of our rate modifications have little effect on most calculated results for all but the earliest times (Shalabiea et al. 1998).

Although the Monte Carlo method has not yet been successfully integrated into a time dependent code, it can be tested for complex systems at steady state. Specifically, following Tielens \& Hagen (1982), one can use a purely gas phase model to determine steady-state gaseous abundances and, assuming these abundances to be fixed, one can determine mole fractions of surface species by both rate equations and Monte Carlo techniques.

Eric Herbst would like to acknowledge the NSF for support of his research in astrochemistry. He is also grateful to the Ohio Supercomputer Center for computer time on their Cray Y-MP/8. P. C. acknowledges support from ASIgrant 94-RS-152, ASI-grant ARS-96-66, and CNR-grant 96/00317.

\section{REFERENCES}

Brown, P. D., \& Charnley, S. B. 1990, MNRAS, 244, 432

Charnley, S. B., Tielens, A. G. G. M., \& Rodgers, S. D. 1997, ApJ, 482, L203

Hasegawa, T. I., \& Herbst, E. 1993, MNRAS, 261, 83

Hasegawa, T. I., Herbst, E., \& Leung, C. M. 1992, ApJS, 82, 167

Pickles, J. B., \& Williams, D. A. 1977, Ap\&SS, 52, 443

Shalabiea, O. M., Caselli, P., \& Herbst, E. 1998, ApJ, submitted
Shalabiea, O. M., \& Greenberg, J. M. 1994, A\&A, 290, 266

Somorjai, G. A. 1994, Introduction to Surface Chemistry and Catalysis (New York: Wiley)

Tielens, A. G. G. M., \& Allamandola, L. J. 1987, in Interstellar Processes, ed. D. J. Hollenbach \& H. A. Thronson, Jr. (Dordrecht: Reidel), 397

Tielens, A. G. G. M., \& Hagen, W. 1982, A\&A, 114, 245 